\title{
PBOV1 correlates with progression of ovarian cancer and inhibits proliferation of ovarian cancer cells
}

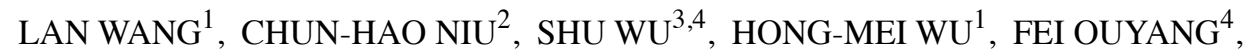 \\ MIAN $\mathrm{HE}^{4}$ and SHAN-YANG HE

\begin{abstract}
${ }^{1}$ Department of Pathogen Biology and Immunology, School of Basic Courses, Guangdong Pharmaceutical University, Guangzhou, Guangdong 510006; ${ }^{2}$ Department of Collaborative Innovation Center for Cancer Medicine,

${ }^{3}$ State Key Laboratory of Oncology in South China, Guangzhou, Guangdong 510060; ${ }^{4}$ Department of Obstetrics and

Gynecology, The First Affiliated Hospital, Sun Yat-Sen University, Guangzhou, Guangdong 510700, P.R. China
\end{abstract} \\ State Key Laboratory of Oncology in South China, Sun Yat-Sen University Cancer Center, Guangzhou, Guangdong 510060;
}

Received July 25, 2015; Accepted October 5, 2015

DOI: $10.3892 /$ or.2015.4396

\begin{abstract}
Prostate and breast cancer overexpressed 1 (PBOV1) is significantly upregulated in prostate, breast and bladder cancer, while its expression status in ovarian cancer and its clinical significance are unclear. We examined the expression levels of PBOV1 mRNA and protein in ovarian cancer cell lines and primary tissues using real-time PCR and western blotting. Immunohistochemistry was employed to analyze PBOV1 expression in 17 normal ovaries, 13 cystadenoma tissues, 14 borderline tumor tissues, and 165 clinicopathologically characterized ovarian cancers. There was negative PBOV1 expression in the 17 normal ovarian epithelial tissues. Compared to the normal ovarian epithelial cells, PBOV1 mRNA and protein were overexpressed in ovarian cancer cell lines. There was high PBOV1 protein expression in the ovarian cancer tissues from 59 of the 165 (35.8\%) patients; PBOV1 expression was weak in $106(64.2 \%)$ patients. Notably, there were significant negative associations between high PBOV1 expression and ascending histological grade, late $\mathrm{pT} / \mathrm{pN} / \mathrm{pM}$, and International Federation of Gynecology and Obstetrics (FIGO) stage $(\mathrm{P}<0.05)$. Patients with high $\mathrm{PBOV} 1$ expression had longer overall survival; patients with low PBOV1 expression had shorter survival. Multivariate analysis revealed that $P B O V 1$ upregulation is an independent prognostic indicator for ovarian cancer and might serve as a tumor-suppressor gene.
\end{abstract}

Correspondence to: Dr Shan-Yang He, Department of Obstetrics and Gynecology, The First Affiliated Hospital, Sun Yat-Sen University, Guangzhou, Guangdong 510700, P.R. China

E-mail: 846645843@qq.com

Dr Lan Wang, Department of Pathogen Biology and Immunology, School of Basic Courses, Guangdong Pharmaceutical University, Guangzhou, Guangdong 510006, P.R. China

E-mail: wanglan3435@hotmail.com

Key words: PBOV1, ovarian cancer, proliferation
Furthermore, PBOV1 overexpression inhibited ovarian cancer cell proliferation and tumorigenesis in vitro and in a tumor transplantation nude mouse model. In conclusion, our results suggest that PBOV1 may play an important role in suppressing ovarian cancer proliferation and carcinogenesis. PBOV1 may be a novel and useful prognostic marker and potential target for treating human ovarian cancer.

\section{Introduction}

Ovarian cancer is a common lethal gynecological malignancy worldwide, causing over 140,000 deaths every year $(1,2)$. Although improved debulking surgery and the introduction of platinum-taxane regimens have been applied in its treatment, the 5-year survival rate is $\sim 40 \%$ (3). This poor prognosis is mostly related to late diagnosis; approximately $70 \%$ of patients are diagnosed at the advanced stages of ovarian cancer [International Federation of Gynecology and Obstetrics (FIGO) III/IV] $(2,4)$. It is difficult to diagnose ovarian cancer at its early stages (FIGO I/II) before it spreads and advances to later stages (FIGO III/IV), as the majority of symptoms are non-specific and thus of little use in the diagnosis at the early stage (4). Serum molecular tests such as cancer antigen (CA) 125 test are useful in differential diagnosis, but have not been demonstrated as effective methods for screening for early-stage ovarian cancer due to the unacceptable low sensitivity and specificity (5). It has been considered that numerous other genetic changes are involved in the development and progression of ovarian cancer $(6,7)$. However, little is known concerning the exact molecular events leading to its development and progression. Therefore, further understanding of the molecular mechanism and discovering valuable diagnostic markers as well as novel therapeutic strategies are major challenges in ovarian cancer.

Prostate and breast cancer overexpressed 1 (PBOV1, or $U R O C 28$ or $U C 28$ ), a human protein-coding gene with a 135-amino acid open reading frame, is overexpressed in prostate, breast and bladder cancer, but has not been demonstrated as being overexpressed in lung and colon cancer tissues (8). PBOV1 mRNA and protein were found to be upregulated in 
glandular epithelial cells of prostate cancer, and were regulated by androgen treatment (9). Compared with normal individuals, patients with prostate cancer had higher serum PBOV1 protein levels. In breast cancer cells, frequently overexpressed PBOV1 was downregulated by estradiol in a dose-dependent manner (9). Fluorescence in situ hybridization showed that there were increased copy numbers of the gene loci encoding PBOV1 in $67 \%$ of prostate tumor foci (10). Another group suggested that the PBOV1 rs6927706 polymorphism may be a risk factor for breast cancer (11). Using comparative genomics analysis, Samusik et al showed that the PBOV1 protein-coding sequence is $80 \%$ unique to humans and originated de novo during primate evolution through a series of frame-shift and stop codon mutations. They found that $P B O V 1$ is expressed in multiple tumor types and higher levels of PBOV1 expresion positively correlate with relapse-free survival in breast cancer (12). As with breast and prostate cancer, ovarian cancer is closely linked to sex hormones. Therefore, fully elucidating the status of PBOV1 expression and its clinical/prognostic relevance in ovarian cancer is warranted.

In the present study, we report, for the first time, the characterization of PBOV1 expression in human ovarian cancer tissues and its correlation with clinicopathological features. PBOV1 expression was negatively correlated with ovarian cancer FIGO stage, T/N/M classification, and growth. The effectiveness of PBOV1 as an independent prognostic factor was assessed using multivariate analysis. Our results strongly suggest that $P B O V 1$ could be a potential promising biomarker for predicting the prognosis of patients with ovarian cancer, serve as a tumor-suppressor gene, and may be a potential target for ovarian cancer therapy.

\section{Materials and methods}

Patients and tissue specimens. A total of 209paraffin-embedded epithelial ovarian specimens, comprising 17 normal ovarian tissues [also scratched and used as control in real-time reverse transcription (RT)-PCR and western blotting], 13 cystadenoma tissues, 14 borderline tumor tissues, and 165 invasive carcinoma tissues, were obtained from paraffin-embedded tissues archived between 1996 and 2007 and histopathologically diagnosed at the Department of Pathology, Cancer Center, Sun Yat-Sen University (Guangzhou, China). The 17 normal ovary specimens were obtained from excise for non-ovarian diseases. Four non-cancerous ovarian tissues (N1-N4) and five ovarian cancer tissues (T1-T5) were obtained by resection from 9 different patients with ovarian cancer at the Cancer Center of Sun Yat-Sen University. All tissues were pathologically characterized, and Table I summarizes the clinical information concerning the ovarian cancer samples. None of the cancer patients in this study had received preoperative radiation or chemotherapy. The use of the clinical specimens was approved by the Local Institutional Review Board and the Ethics Committee of the Sun Yat-Sen University Cancer Center (Guangzhou, Guangdong, China), and conformed to the ethical guidelines of the Helsinki Declaration.

Cell culture. Primary normal breast epithelial cells (NBECs) were purification and cultured from the excised tissue from a 30-year-old woman with breast plastic surgery at the First
Table I. Association of PBOV1 expression with clinicopathological features of the ovarian carcinoma cases.

\begin{tabular}{|c|c|c|c|c|}
\hline & \multirow[b]{2}{*}{$\begin{array}{c}\text { All } \\
\text { cases }\end{array}$} & \multicolumn{2}{|c|}{ PBOV1 protein } & \multirow[b]{2}{*}{ P-value } \\
\hline & & $\begin{array}{c}\text { Low } \\
\text { expression }\end{array}$ & $\begin{array}{c}\text { High } \\
\text { expression }\end{array}$ & \\
\hline \multicolumn{5}{|c|}{$\begin{array}{l}\text { Age at surgery } \\
\text { (years) }\end{array}$} \\
\hline$\leq 47.6$ & 74 & 44 & 30 & 0.25 \\
\hline$>47.6$ & 91 & 62 & 29 & \\
\hline \multicolumn{5}{|c|}{ Histological type } \\
\hline Serous & 95 & 65 & 30 & 0.333 \\
\hline Mucinous & 36 & 20 & 16 & \\
\hline Others & 34 & 21 & 13 & \\
\hline \multicolumn{5}{|c|}{$\begin{array}{l}\text { Histological grade } \\
\text { (Silverberg) }\end{array}$} \\
\hline $\mathrm{G} 1$ & 15 & 7 & 8 & 0.01 \\
\hline G2 & 94 & 56 & 38 & \\
\hline G3 & 56 & 43 & 13 & \\
\hline \multicolumn{5}{|l|}{ pT status } \\
\hline pT1 & 23 & 9 & 14 & $<0.001$ \\
\hline pT2 & 25 & 10 & 15 & \\
\hline pT3 & 117 & 87 & 30 & \\
\hline \multicolumn{5}{|l|}{ pN status } \\
\hline $\mathrm{pN} 0$ & 53 & 27 & 26 & 0.014 \\
\hline pN1 & 111 & 79 & 32 & \\
\hline \multicolumn{5}{|l|}{ pM status } \\
\hline $\mathrm{pMX}$ & 129 & 77 & 52 & 0.021 \\
\hline pM1 & 36 & 29 & 7 & \\
\hline \multicolumn{5}{|l|}{ FIGO stage } \\
\hline I & 23 & 9 & 14 & $<0.001$ \\
\hline II & 14 & 4 & 10 & \\
\hline III & 92 & 64 & 28 & \\
\hline IV & 36 & 29 & 7 & \\
\hline
\end{tabular}

Affiliated Hospital of Sun Yat-Sen University (China). Primary NBECs and the ovarian cancer cell lines were maintained according to our previous study (13).

RNA extraction and real-time quantitative PCR. Total RNA from cultured cells was extracted using TRIzol reagent (Invitrogen) according to the manufacturer's instructions. Complementary DNA (cDNA) was amplified and quantified using an ABI Prism 7500 Sequence Detection system (Applied Biosystems, Foster City, CA, USA) and SYBR Green I (Molecular Probes, Invitrogen). The primers used were as follows: $P B O V 1$ forward, 5'-TGAGTCCCCTCTCGGTA ATG-3' and reverse, 5'-GCCCCGAGTTAAGAACATCA-3'. Expression data were normalized to the geometric mean of the housekeeping gene glyceraldehyde-3-phosphate dehydrogenase $(G A P D H)$ to control variability in expression levels (forward, 5'-ACCACAGTCCATGCCATCAC-3' and reverse, 5'-TCCACCACCCTGTTGCTGTA-3'), and calculated as 
$2^{-[(\mathrm{CT} \text { of } P B O V I)-(\mathrm{CT} \text { of } G A P D H)]}$, where CT represents the threshold cycle for each transcript.

Plasmid and transfection. The full-length sequence of PBOVI is 408-bp long and was cloned into a pSin plasmid (Promega, Madison, WI, USA). Retroviral production and infection were performed as previously described (14).

Western blotting. Western blotting was performed according to standard methods as previously described, using anti-PBOV1 and anti-Ki67 (Santa Cruz Biotechnology, Santa Cruz, CA, USA) (13). Each sample was detected and analyzed three times.

Immunohistochemistry (IHC). The IHC procedure and PBOV1 expression scoring were performed as previously described (15). The proportion of positively stained tumor cells was graded as follows: 0 (no positive tumor cells), 1 ( $<10 \%$ positive tumor cells), 2 (10-50\% positive tumor cells), and $3(>50 \%$ positive tumor cells). Each staining intensity was scored on a scale of 0 (no staining), 1 (weak staining, light yellow), 2 (moderate staining, yellowish brown), and 3 (strong staining, brown). The staining index was calculated as in our previous study (13). Cut-off values for defining high and low PBOV1 expression were selected based on a measure of heterogeneity with log-rank test statistics with respect to overall survival, and an optimal cut-off value was identified. The staining index scores $>6$ and $<4$ were used to define tumors with high and low PBOV1 expression, respectively. The AxioVision Rel.4.6 computerized image analysis system assisted by an automated measurement program (Carl Zeiss, Oberkochen, Germany) was used for quantitative analysis of the IHC staining.

\section{3-(4,5-Dimethyl-2-thiazolyl)-2,5-diphenyl-2H-tetrazolium} bromide (MTT) assay. Cells were cultured in 96-well plates (1x10 $/$ well). At various time-points, $0.5 \mathrm{mg} / \mathrm{ml} \mathrm{MTT} \mathrm{was}$ used to treat cells for $4 \mathrm{~h}$ at $37^{\circ} \mathrm{C}$. The medium was removed, and $150 \mu \mathrm{l}$ dimethyl sulphoxide (DMSO; Sigma-Aldrich) was added and then absorbance values were measured. All experiments were performed in triplicate as previously reported (16).

Colony formation assay. Cells (1,000/plate) were incubated in 6 -well plates for 10 days. The colonies were stained with $1.0 \%$ crystal violet for $30 \mathrm{sec}$, followed by 5 -min fixation in $10 \%$ formaldehyde.

Anchorage-independent growth assay. Cells [500 in $2 \mathrm{ml}$ complete medium plus $0.3 \%$ agar (Sigma-Aldrich)] were seeded in 6-well plates. All experiments were performed in triplicate for each cell line according to our previous study (16).

Xenograft tumor model. BALB/c-nu mice (4-5 weeks old, 18-20 g) were purchased from Hunan SJA Laboratory Animal Co., Ltd. (Changsha, Hunan, China). The Institutional Animal Care and Use Committee of Sun Yat-Sen University approved all experimental procedures. Each mouse was subcutaneously injected in situ with SKOV3-vector cells $\left(5 \times 10^{6}\right)$ on the left side and with SKOV3-PBOV1 cells $\left(5 \times 10^{6}\right)$ on the right side. Tumors were examined every five days; length (L) and width (W) were measured using calipers, and tumor volumes were calculated using the equation $\left(\mathrm{Lx} \mathrm{W}^{2}\right) / 2$. On day 40 , the animals were euthanized, and the tumors were excised and weighed.

Statistical analysis. All statistical analyses were carried out using the SPSS v. 13.0 statistical software packages. The relationship between PBOV1 expression and clinicopathological characteristics was analyzed using the Chi-square test. Bivariate correlations between study variables were calculated by Spearman's rank correlation coefficients. Survival curves were plotted using the Kaplan-Meier method and compared using the log-rank test. Survival data were evaluated using univariate and multivariate Cox regression analyses. A p-value $<0.05$ was considered to indicate a statistically significant result in all cases.

\section{Results}

PBOV1 upregulation in ovarian cancer cell lines. Representative overexpression of PBOV1 has been reported in breast and prostate cancer (12). Its expression status in ovarian cancer, however, remains unclear. To determine PBOV1 protein expression, western blotting was performed using protein samples from human normal ovarian tissues, NBECs, and ovarian cancer cell lines (OVCAR4, SKOV3 COV644, OV56, TOV-21G, OV90). To investigate whether PBOV1 was upregulated at the transcription level, $P B O V 1$ mRNA was quantified using real-time PCR. Both PBOV1 protein and mRNA expression were markedly upregulated in the ovarian cancer cell lines (Fig. 1A and B).

PBOV1 upregulation in ovarian cancer tissues. To determine whether PBOV1 upregulation in ovarian cancer cell lines is clinically correlated with ovarian cancer progression, realtime PCR analysis and western blotting were performed using non-cancerous ovarian tissues from four patients with other ovarian diseases and ovarian cancer tissues from 5 patients with ovarian cancer. Both PBOV1 mRNA and protein levels were differentially overexpressed in the primary ovarian cancer samples (Fig. 1C and D). Quantification determined that all five tumors had >3-fold increased PBOV1 mRNA compared with the normal tissues (Fig. 1D).

IHC staining of PBOV1 protein in human ovarian tissues. To investigate PBOV1 upregulation in ovarian cancer, we further examined PBOV1 expression in 165 paraffin-embedded, archived ovarian tissues, and found that PBOV1 protein was located in the cytoplasm and cell membrane. As shown in Fig. 2, there was negative PBOV1 expression in normal ovarian surface epithelium (Fig. 2A; detected in none of the 17 samples), cystadenoma (Fig. 2B; detected in 1 of the 13 samples), and borderline tumors (Fig. 2C; detected in 2 of the 14 samples; Table II). Although PBOV1 was upregulated in the tumor tissues, the expression levels differed. In the 165 patients with ovarian cancer, there was high PBOV1 expression in $35.8 \%$ of the samples (Fig. 2D-F) and low expression in $64.2 \%$ of the samples (Fig. 2G-I). There was differential expression of PBOV1 protein between the types of ovarian cancer, such as serous ovarian cancer (Fig. 2E and H) and mucinous ovarian cancer (Fig. $2 \mathrm{~F}$ and I). Taken together, 
A

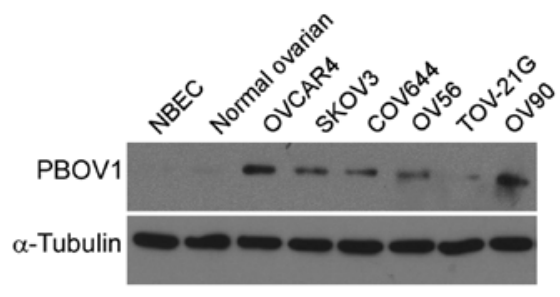

C

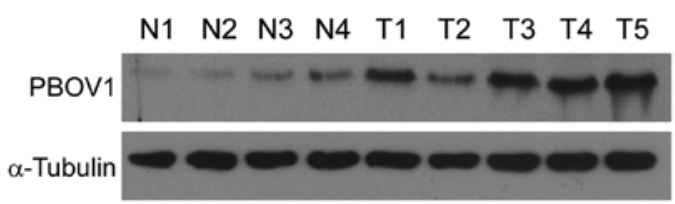

B

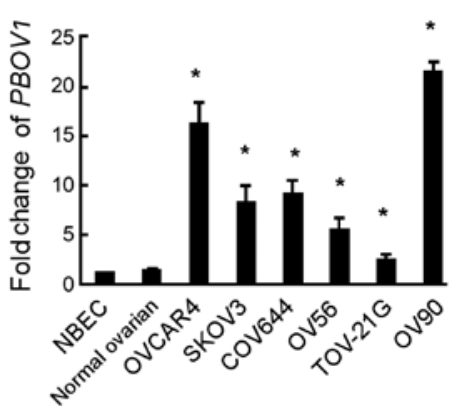

D

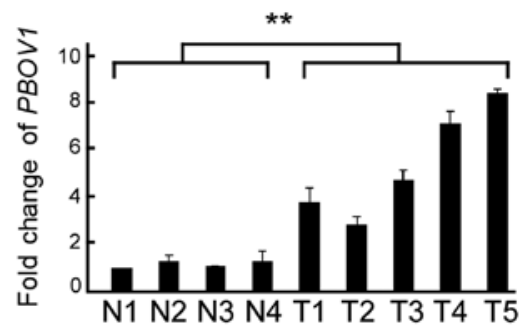

Figure 1. Expression analysis of PBOV1 protein and mRNA in ovarian epithelial cells and tissues. (A) Western blot assay of PBOV1 protein expression in normal ovarian epithelial cells (normal ovarian) and ovarian cancer cell lines. Primary normal breast epithelial cells (NBECs) were used as the negative control. (B) PBOV1 mRNA expression in NBECs, normal ovarian epithelial cells, and ovarian cancer cell lines. (C) Western blot analysis of PBOV1 protein expression in normal ovarian epithelial cells from 4 patients without ovarian cancer (N1-N4) and primary ovarian tumors from 5 patients with ovarian cancer (T1-T5). $\alpha$-tubulin was used as the loading control. (D) Real-time PCR analysis of PBOV1 expression in fresh tissues. GAPDH was used the loading control. ${ }^{*} \mathrm{P}<0.05,{ }^{* *} \mathrm{P}<0.001$
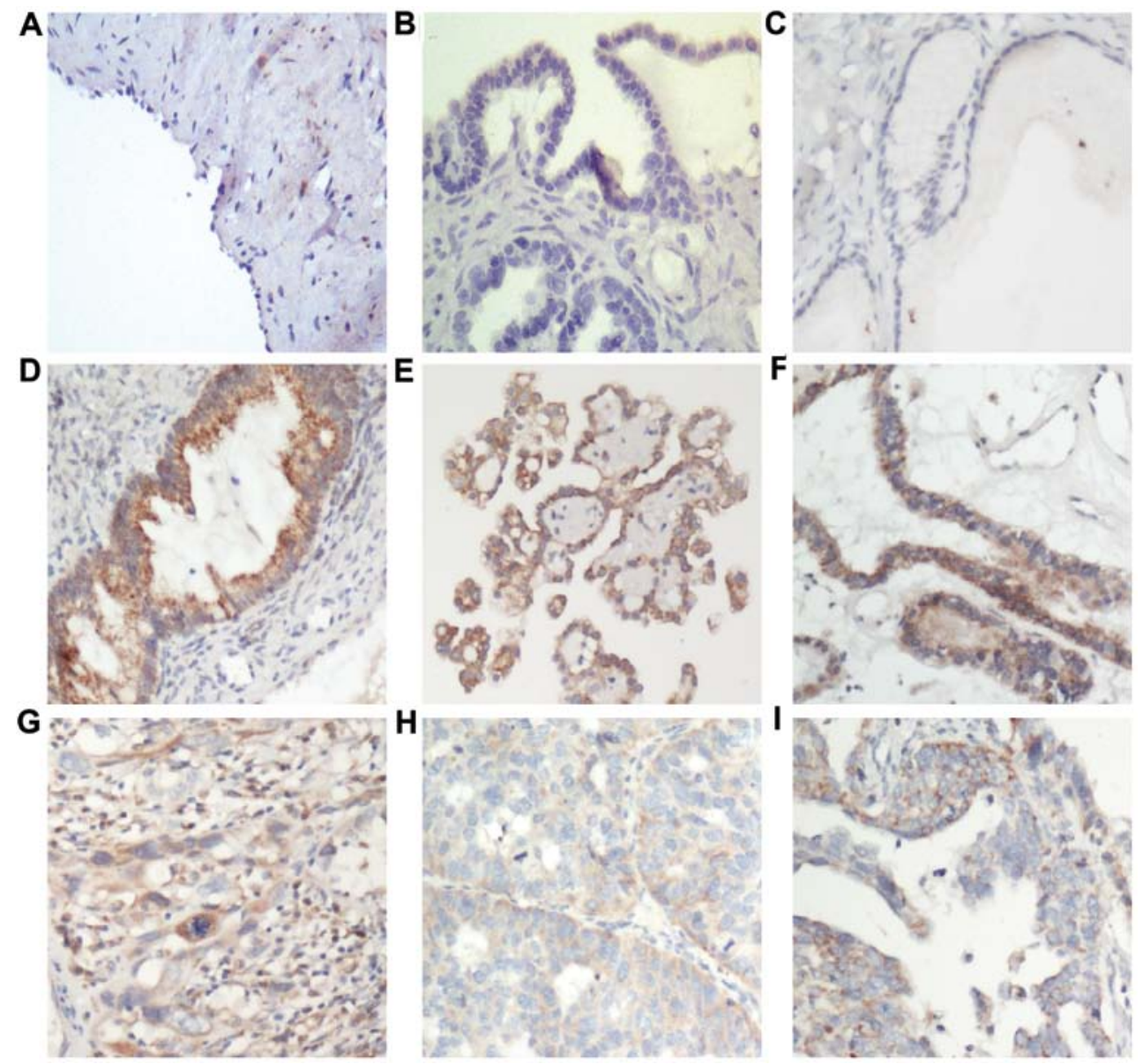

Figure 2. IHC staining of PBOV1 protein in human ovarian tissues. (A) Negative expression of PBOV1 in normal ovarian surface epithelium (x200 magnification). (B) Ovarian cystadenoma with negative expression of PBOV1 in 90\% of tumor cells (x200 magnification). (C) Negative expression of PBOV1 in borderline ovarian tumors (x200 magnification). (D-F) Intense expression of PBOV1 in three early FIGO stage ovarian carcinoma samples; $>90 \%$ of carcinoma cells had strong positive staining for PBOV1 (x200 magnification). (G-I) Three ovarian carcinoma samples with low PBOV1 expression (x200 magnification). 
Table II. PBOV1 expression in normal ovaries and in benign and malignant epithelial ovarian tumors.

PBOV1 protein

\begin{tabular}{lcccc} 
Samples & All cases & High expression $(\%)$ & Low expression $(\%)$ & Negative expression (\%) \\
\cline { 3 - 5 } Normal ovaries & 17 & $0(0.0)$ & $0(0.0)$ & $17(100.0)$ \\
Cystadenomas & 13 & $0(0.0)$ & $1(7.7)$ & $12(92.3)$ \\
Borderline tumors & 14 & $0(0.0)$ & $2(16.7)$ & $12(83.3)$ \\
Invasive carcinomas & 165 & $59(35.8)$ & $106(64.2)$ & $0(0)$
\end{tabular}

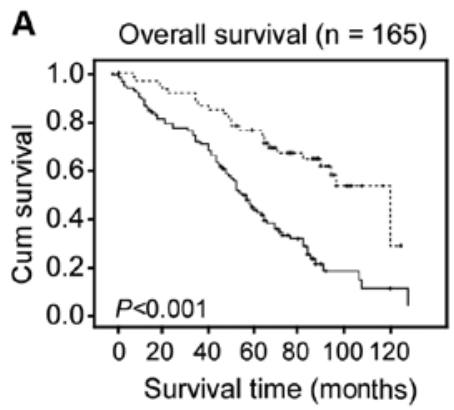

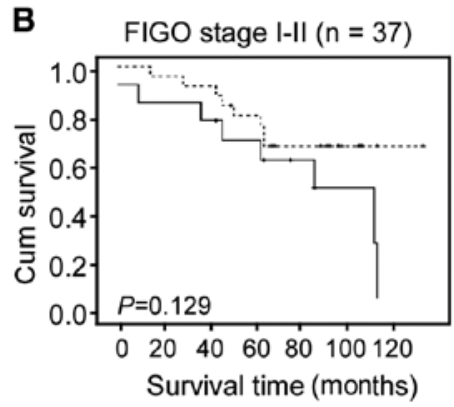

- Low expression of PBOV1

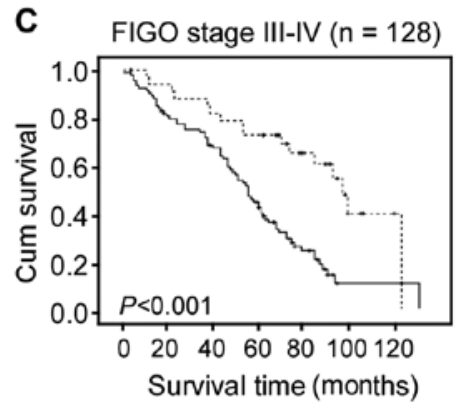

--- High expression of PBOV1

Figure 3. Kaplan-Meier curves with univariate analyses (log-rank) of patients with ovarian tumors. (A) Among 165 patients with ovarian tumors, the cumulative 5-year survival rate was $37.5 \%$ (95\% confidence interval, $0.283-0.467$ ) in the low-PBOV1 protein expression group ( $\mathrm{n}=106$ ); the rate was $78.8 \%$ (95\% confidence interval, 0.682-0.894) in the high-expression group ( $\mathrm{n}=59)$. (B) Statistical significance of the difference between curves of intense PBOV1 expression $(n=24)$ and low PBOV1 expression $(n=13)$ when patients with clinical stage I-II disease were compared. (C) Statistical significance of the difference between curves of intense PBOV1 expression $(n=35)$ and low PBOV1 expression $(n=103)$ when patients with clinical stage III-IV disease were compared. P-values were calculated by log-rank testing.

these observations suggest that PBOV1 expression is a common feature of ovarian cancer.

Association of PBOVI expression with ovarian cancer clinicopathological features. We studied the association between PBOV1 expression in ovarian cancer and several known clinicopathological features. PBOV1 expression was negatively correlated with histological grade $(\mathrm{P}=0.01)$, pT status $(\mathrm{P}<0.001), \mathrm{pN}$ status $(\mathrm{P}=0.014), \mathrm{pM}$ status $(\mathrm{P}<0.021)$, and FIGO stage $(\mathrm{P}<0.001)$. There was no significant correlation between PBOV1 expression and histological type or patient age at surgery $(P>0.05$, Table I). Taken together, these observations support the notion that ovarian cancer progression is associated with decreased PBOV1 expression.

Association between PBOVI expression and survival. The statistical analysis findings in Table IV revealed a positive correlation between the PBOV1 level and patient survival $(\mathrm{P}<0.001)$. In conjunction with PBOV1 protein expression, established prognostic predictors of patient survival, including histological grade, $\mathrm{pT} / \mathrm{pN} / \mathrm{pM}$ status, and FIGO stage, were evaluated with Kaplan-Meier analysis and the log-rank test. As shown in Fig. 3, survival was significantly different between patients with low and high PBOV1 expression $(\mathrm{P}<0.001)$ : patients with high PBOV1 expression had a longer overall survival. The cumulative 5-year survival rate in the high-PBOV1 expression group was $86 \%$; in the low-PBOV1 expression group, it was $63 \%$ (Fig. 3A). Univariate and multi- variate analyses were used to determine whether PBOV1 expression level is an independent prognostic factor of patient outcome. Tables III and IV showed that PBOV1 expression, as well as FIGO stage and histological grade, were independent prognostic factors. Furthermore, the prognostic value of PBOV1 expression in specific patient subgroups was evaluated according to clinical staging. Despite the difference in overall survival length between the low- and high-PBOV1 expression groups, overall survival did not differ in the early clinical subgroups (stages I and II, $n=37$; log-rank, $\mathrm{P}<0.001$; Fig. 3B). In the advanced disease group (stages III and IV, $n=128$ ), patients with high PBOV1 expression had significantly higher overall survival rates compared with those with low PBOV1 expression ( $\mathrm{P}<0.001$; Fig. 3C). Taken together, our data suggest that PBOV1 may represent a novel and potentially useful independent prognostic biomarker for patients with ovarian cancer.

Overexpression of PBOV1 suppresses ovarian cancer cell proliferation. To investigate the biological role of PBOV1 in ovarian cancer progression, SKOV3 and OVCAR4 ovarian cancer cells were transduced to stably overexpress PBOV1 (Fig. 4A). The expression of Ki67, an acknowledged marker of proliferation, was found to be downregulated in the PBOV1-overexpressing cells (Fig. 4A). MTT and colony formation assays showed that PBOV1 overexpression markedly reduced the growth rate of the SKOV3 and OVCAR4 cells compared with that of the vectortransduced cells (Fig. 4B and C). Moreover, ectopic expression 
Table III. Univariate survival analysis (log-rank test) of the clinicopathological parameters and PBOV1 expression in the prognosis of 164 patients with ovarian carcinoma.

\begin{tabular}{|c|c|c|c|c|}
\hline Variable & $\begin{array}{c}\text { All } \\
\text { cases }\end{array}$ & $\begin{array}{c}\text { Mean } \\
\text { survival } \\
\text { (months) }\end{array}$ & $\begin{array}{c}\text { Median } \\
\text { survival } \\
\text { (months) }\end{array}$ & P-value \\
\hline \multicolumn{5}{|c|}{$\begin{array}{l}\text { Age at surgery } \\
\text { (years) }\end{array}$} \\
\hline$\leq 47.6$ & 74 & 76.7 & 67.4 & 0.323 \\
\hline$>47.6$ & 91 & 63.4 & 61.9 & \\
\hline \multicolumn{5}{|c|}{ Histological type } \\
\hline Serous & 95 & 67.3 & 58.6 & 0.157 \\
\hline Mucinous & 36 & 82.6 & 91.7 & \\
\hline Others & 34 & 69.3 & 84.0 & \\
\hline \multicolumn{5}{|c|}{$\begin{array}{l}\text { Histological grade } \\
\text { (Silverberg) }\end{array}$} \\
\hline G1 & 15 & 97.8 & 108.4 & $<0.001$ \\
\hline $\mathrm{G} 2$ & 94 & 71.4 & 74.1 & \\
\hline G3 & 56 & 51.6 & 48.6 & \\
\hline \multicolumn{5}{|l|}{ pT status } \\
\hline pT1 & 23 & 82.5 & 108.4 & 0.003 \\
\hline pT2 & 25 & 87.1 & - & \\
\hline pT3 & 117 & 65.2 & 59.7 & \\
\hline \multicolumn{5}{|l|}{ pN status } \\
\hline pNO & 53 & 84.7 & 108.4 & 0.006 \\
\hline pN1 & 112 & 65.4 & 59.7 & \\
\hline \multicolumn{5}{|l|}{ pM status } \\
\hline $\mathrm{pMX}$ & 129 & 75.8 & 74.1 & 0.005 \\
\hline pM1 & 36 & 55.9 & 58.6 & \\
\hline \multicolumn{5}{|l|}{ FIGO stage } \\
\hline I & 23 & 82.4 & 108.4 & $<0.001$ \\
\hline II & 14 & 102.6 & - & \\
\hline III & 92 & 67.9 & 68.5 & \\
\hline IV & 36 & 55.9 & 58.6 & \\
\hline \multicolumn{5}{|c|}{ PBOV1 expression } \\
\hline Low & 106 & 57.6 & 55.3 & $<0.001$ \\
\hline High & 58 & 99.2 & 121.6 & \\
\hline
\end{tabular}

of PBOV1 significantly reduced SKOV3 and OVCAR4 cell anchorage-independent growth, as indicated by the decreased colony number and size (Fig. 4D).

In vivo assay reveals the suppressive effect of PBOVI on tumorigenicity. To validate the in vitro cell proliferation assay results, we performed in vivo assays to evaluate the tumorigenic effect of PBOV1 in non-obese diabetic/severe combined immunodeficiency (NOD/SCID) mice using the SKOV3 cell line. PBOV1-transfected cells exhibited an anti-proliferative tendency in the nude mice (Fig. 5A and B). The PBOV1 expression of the excised tumors was verified by western blotting (Fig. 5C). Our results demonstrated that PBOV1 plays an important role in the tumorigenicity of ovarian cancer in vivo.
Table IV. Multivariate analysis of overall survival (Cox regression model).

\begin{tabular}{lccr}
\hline Variable & Relative risk & $\begin{array}{c}95 \% \text { confidence } \\
\text { interval }\end{array}$ & P-value \\
\hline PBOV1 & 3.017 & $1.771-5.141$ & $<0.001$ \\
FIGO stage & 0.65 & $0.401-1.055$ & 0.036 \\
Histological grade & 0.519 & $0.335-0.804$ & 0.005 \\
\hline
\end{tabular}

\section{Discussion}

The key finding of our study is that PBOV1 upregulation, a common molecular change in human ovarian cancer, and PBOV1 overexpression inhibit ovarian cancer cell proliferation and tumorigenicity. Our study identifies a close correlation between PBOV1 downregulation and disease progression as well as poor patient survival. The in vitro and in vivo assays both demonstrated the suppressive role of PBOV1 on ovarian cancer cells. Our findings suggest that PBOV1 can potentially be targeted as a therapeutic strategy for ovarian cancer.

Ovarian, breast, endometrial and prostate cancer are hormone-dependent cancers regulated by hormones such as estrogen, progesterone, or androgen (17-20). Based on hormonal responsiveness, the development of effective prevention strategies for breast, endometrial, ovarian and prostate cancer is of paramount importance in the care of patients at risk for these malignancies (21-23). Estrogen regulates proteases and antiproteases in both ovarian and breast cancer cells $(24,25)$. These studies indicate that the study direction of ovarian cancer can be traced from breast and prostate cancer. It was previously reported that PBOV1 expression is upregulated in breast and prostate cancer cells and is positively regulated by estrogen and dihydrotestosterone, respectively $(9,10)$. A previous study stated that PBOV1 is expressed in human ovarian serous cystadenocarcinoma, and the expression level and function of PBOV1 protein in ovarian cancer are unknown (12). We found that, in comparison with that in normal ovarian cells and tissues, both PBOV1 mRNA and protein were upregulated in ovarian cancer cell lines and clinical tumors. The conclusion obtained from the above research that $P B O V 1$ emerged de novo as a protein-coding gene, as reported by Samusik et al supported our current data that PBOV1 is upregulated in ovarian cancer (12). However, how PBOV1 is upregulated in ovarian cancer requires further exploration.

The low PBOV1 protein expression in advanced FIGO stages is of great interest, and PBOV1 correlates inversely with clinical advancement and shorter survival in breast cancer. We noted that expression was intense in samples obtained from low FIGO stage patients and was lower in samples from high FIGO stage patients. PBOV1 protein expression levels were significantly correlated with the prognosis of ovarian cancer, where low PBOV1 protein expression in ovarian cancer lesions was closely associated with advanced FIGO staging; higher T, $\mathrm{N}$, and M classification; histological grade (Silverberg); and shorter survival. It was previously reported that higher levels of PBOV1 significantly correlated with relapse-free survival, which could only be observed in patients with lymph node 
A

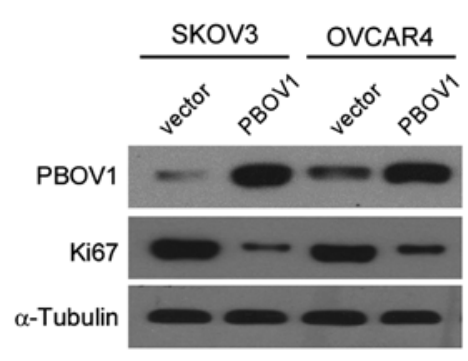

C

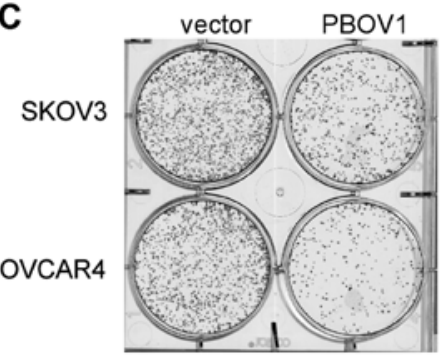

D

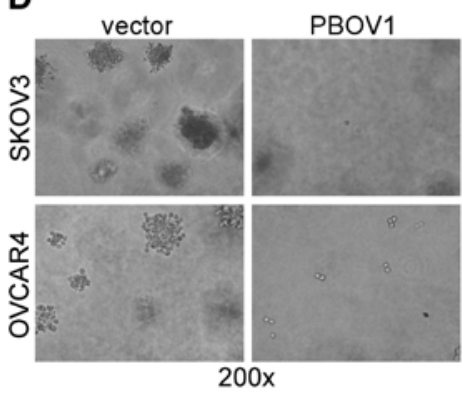

B
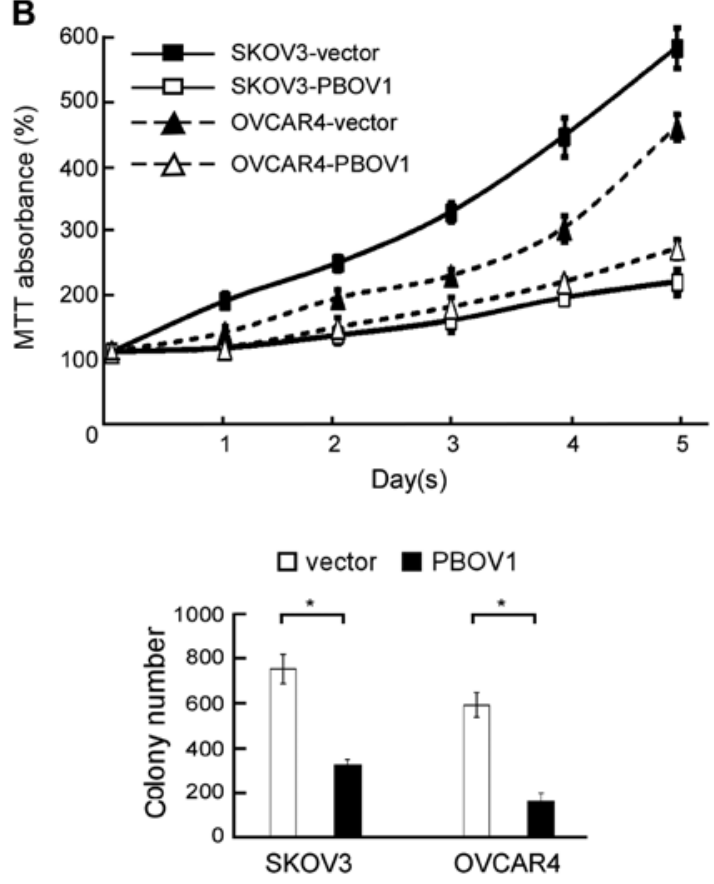

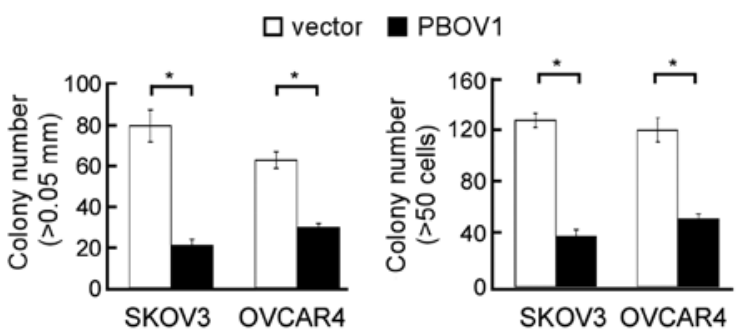

Figure 4. Overexpression of PBOV1 suppresses SKOV3 and OVCAR4 cell proliferation and tumorigenicity. (A) Western blot analysis of increased PBOV1 expression and decreased Ki67 expression. (B) MTT assay showed that PBOV1-transfected cells grew slower than the vector-transfected cells. (C) Representative micrographs (left) and quantification of crystal violet-stained cell colonies. (D) Representative micrographs (left) and colony numbers in the anchorageindependent growth assay. Each bar represents the mean of three independent experiments. ${ }^{*} \mathrm{P}<0.05$.

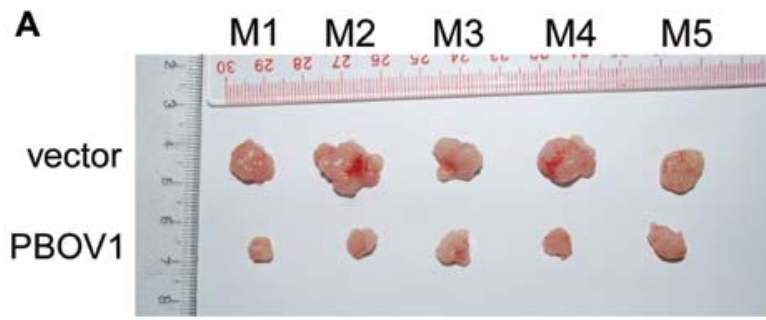

B

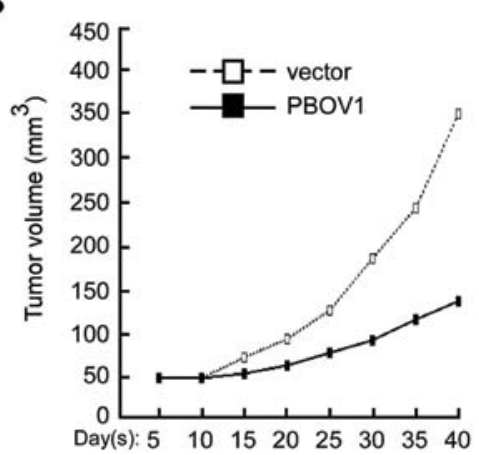

C

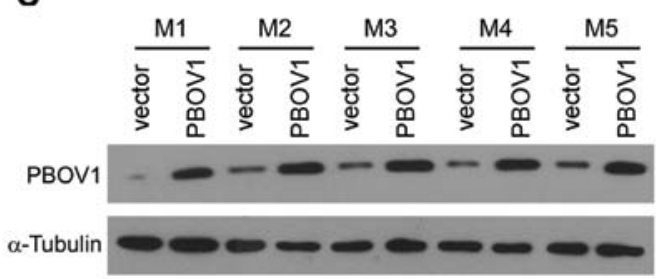

Figure 5. In vivo assays of the inhibitory effect of PBOV1 on cell proliferation. (A) Excised tumors 40 days after cell injection in NOD/SCID mice (M1-M5). (B) Tumor growth curves measured every five days. (C) Western blot analysis of PBOV1 protein expression in the excised tumors. 
metastasis (8). Analysis of a gene expression dataset of clinical glioma samples showed that tumor samples from patients with proneural glioma who survived for more than 209 weeks had higher PBOV1 expression levels (12). Herein, we believe that PBOV1 protein may act as a tumor suppressor. Its higher expression in stage I/II disease indicates that PBOV1 may be involved in the origin of the tumor and render it helpful for early diagnosis.

To evaluate the biological function of PBOV1 in ovarian cancer, we constructed PBOV1-overexpressing cell models. The proliferation inhibitory activity of PBOV1 was demonstrated in the in vitro and in vivo assays. This is the first suggestion that PBOV1 may be involved in suppressing ovarian cancer cell proliferation and tumorigenicity.

Why does PBOV1 overexpression decrease as the degree of malignancy increases? A transcription- or translation-associated molecule may play a major role in regulating PBOV1 in ovarian cancer. MicroRNAs (miRNAs), a class of small non-coding RNAs, inhibit gene translation or facilitate mRNA degradation, resulting in the repression of target gene expression $(26,27)$. Several miRNAs are involved in regulating ovarian cancer, such as miR-22 (28), miR-200 (29), miR-210 (30) and miR-16 (31). By analyzing the 3' untranslated region of $P B O V 1$ in TargetScan Human, we found 10 potential conserved sites targeted by miR-431, miR-132, miR-212 and miR-1299 which require verification in our future research.

PBOV1 protein can be detected in the serum, and higher serum levels of PBOV1 protein were detected in patients with prostate cancer compared with that in normal individuals (9). It would be of great interest to investigate whether such an important marker is also detectable in other patient samples such as blood and ovarian fluid in addition to biopsy or surgical tissues. Confirmation of this would require future large-scale studies. Nevertheless, our study provides a basis for developing a novel diagnostic and prognostic biomarker of ovarian cancer. In summary, our study suggests that PBOV1 overexpression is a common feature in ovarian cancer and may represent a novel predictive marker for the clinical outcome of the disease.

\section{Acknowledgements}

The present study was supported by the National Natural Science Foundation of China (grant no. 81201568).

\section{References}

1. Whitmore SE, Rosenshein NB and Provost TT: Ovarian cancer in patients with dermatomyositis. Medicine (Baltimore) 73: 153-160, 1994.

2. Markman M, Webster K, Zanotti K, Peterson G, Kulp B and Belinson J: Survival following the documentation of platinum and taxane resistance in ovarian cancer: A single institution experience involving multiple phase 2 clinical trials. Gynecol Oncol 93: 699-701, 2004.

3. No authors listed: Debulking surgery in ovarian cancer. J Clin Oncol 4: 1716-1717, 1986

4. Rossing MA, Wicklund KG, Cushing-Haugen KL and Weiss NS: Predictive value of symptoms for early detection of ovarian cancer. J Natl Cancer Inst 102: 222-229, 2010.

5. Chudecka-Głaz AM, Cymbaluk-Płoska AA, Menkiszak JL, Sompolska-Rzechuła AM, Tołoczko-Grabarek AI and Rzepka-Górska IA: Serum HE4, CA125, YKL-40, bcl-2, cathepsin-L and prediction optimal debulking surgery, response to chemotherapy in ovarian cancer. J Ovarian Res 7: 62, 2014.
6. DiSaia PJ and Bloss JD: Treatment of ovarian cancer: New strategies. Gynecol Oncol 90: S24-S32, 2003.

7. Mantha S, Sarasohn D, Ma W, Devlin SM, Chi DS, Roche KL, Suidan RS, Woo K and Soff GA: Ovarian vein thrombosis after debulking surgery for ovarian cancer: Epidemiology and clinical significance. Am J Obstet Gynecol 213: 208.e1-e4, 2015.

8. Krukovskaia LL, Samusik ND, Shilov ES, Polev DE and Kozlov AP: Tumor-specific expression of PBOV1, a new gene in evolution. Vopr Onkol 56: 327-332, 2010 (In Russian).

9. Kamagata C, Tsuji N, Kondoh K, Sasaki M, Kobayashi D, Yagihashi A and Watanabe N: Enhanced expression of the UROC28 gene in human breast cancer: Relationship to ERBB2 gene expression. Anticancer Res 22: 4087-4091, 2002.

10. Doak SH, Jenkins SA, Hurle RA, Varma M, Hawizy A, Kynaston HG and Parry JM: Bone morphogenic factor gene dosage abnormalities in prostatic intraepithelial neoplasia and prostate cancer. Cancer Genet Cytogenet 176: 161-165, 2007.

11. Loizidou MA, Cariolou MA, Neuhausen SL, Newbold RF, Bashiardes E, Marcou Y, Michael T, Daniel M, Kakouri E, Papadopoulos P, et al: Genetic variation in genes interacting with BRCA1/2 and risk of breast cancer in the Cypriot population. Breast Cancer Res Treat 121: 147-156, 2010.

12. Samusik N, Krukovskaya L, Meln I, Shilov E and Kozlov AP: PBOV1 is a human de novo gene with tumor-specific expression that is associated with a positive clinical outcome of cancer. PLoS One 8: e56162, 2013.

13. Li J, Zhang N, Song LB, Liao WT, Jiang LL, Gong LY, Wu J, Yuan J, Zhang HZ, Zeng MS, et al: Astrocyte elevated gene-1 is a novel prognostic marker for breast cancer progression and overall patient survival. Clin Cancer Res 14: 3319-3326, 2008.

14. Hahn WC, Dessain SK, Brooks MW, King JE, Elenbaas B, Sabatini DM, DeCaprio JA and Weinberg RA: Enumeration of the simian virus 40 early region elements necessary for human cell transformation. Mol Cell Biol 22: 2111-2123, 2002.

15. Zhang Z, Li J, Zheng H, Yu C, Chen J, Liu Z, Li M, Zeng M, Zhou F and Song L: Expression and cytoplasmic localization of SAM68 is a significant and independent prognostic marker for renal cell carcinoma. Cancer Epidemiol Biomarkers Prev 18: 2685-2693, 2009.

16. Song L, Wang L, Li Y, Xiong H, Wu J, Li J and Li M: Sam68 up-regulation correlates with, and its down-regulation inhibits, proliferation and tumourigenicity of breast cancer cells. J Pathol 222: 227-237, 2010.

17. Verkooijen HM, Koot VC, Fioretta G, van der Heiden M, Schipper ME, Rapiti E, Peeters PH, Peterse JL and Bouchardy C: Hormone replacement therapy, mammography screening and changing age-specific incidence rates of breast cancer: An ecological study comparing two European populations. Breast Cancer Res Treat 107: 389-395, 2008.

18. Moran-Santa Maria MM, Flanagan J and Brady K: Ovarian hormones and drug abuse. Curr Psychiatry Rep 16: 511, 2014

19. McCullough ML, Patel AV, Patel R, Rodriguez C, Feigelson HS, Bandera EV, Gansler T, Thun MJ and Calle EE: Body mass and endometrial cancer risk by hormone replacement therapy and cancer subtype. Cancer Epidemiol Biomarkers Prev 17: 73-79, 2008.

20. Ansari J, Hussain SA, Zarkar A, Tanguay JS, Bliss J and Glaholm J: Docetaxel chemotherapy for metastatic hormone refractory prostate cancer as first-line palliative chemotherapy and subsequent re-treatment: Birmingham experience. Oncol Rep 20: 891-896, 2008.

21. Markman M, Glass T, Smith HO, Hatch KD, Weiss GR, Taylor SA, Goodwin JW and Alberts DS: Phase II trial of single agent carboplatin followed by dose-intense paclitaxel, followed by maintenance paclitaxel therapy in stage IV ovarian, fallopian tube, and peritoneal cancers: A Southwest Oncology Group trial. Gynecol Oncol 88: 282-288, 2003.

22. Coleman RE: Current and future status of adjuvant therapy for breast cancer. Cancer 97 (Suppl 3): 880-886, 2003.

23. Izadi-Mood N, Sarmadi S and Sanii S: Strategies in the histologic diagnosis of low-grade glandular endometrial neoplasm. Curr Opin Obstet Gynecol 22: 43-50, 2010.

24. Rochefort H, Chalbos D, Cunat S, Lucas A, Platet N and Garcia M: Estrogen regulated proteases and antiproteases in ovarian and breast cancer cells. J Steroid Biochem Mol Biol 76: 119-124, 2001.

25. Elguero S, Patel B and Liu JH: Misperception of estrogen activity in patients treated with an estrogen receptor antagonist. Am J Obstet Gynecol 211: e1-e2, 2014. 
26. Krichevsky AM, King KS, Donahue CP, Khrapko K and Kosik KS: A microRNA array reveals extensive regulation of microRNAs during brain development. RNA 9: 1274-1281, 2003.

27. Kawasaki $\mathrm{H}$ and Taira $\mathrm{K}$ : Hes1 is a target of microRNA-23 during retinoic-acid-induced neuronal differentiation of NT2 cells. Nature 423: 838-842, 2003.

28. Li J, Liang S, Yu H, Zhang J, Ma D and Lu X: An inhibitory effect of miR-22 on cell migration and invasion in ovarian cancer. Gynecol Oncol 119: 543-548, 2010.

29. Hu X, Macdonald DM, Huettner PC, Feng Z, El Naqa IM, Schwarz JK, Mutch DG, Grigsby PW, Powell SN and Wang X: A miR-200 microRNA cluster as prognostic marker in advanced ovarian cancer. Gynecol Oncol 114: 457-464, 2009.
30. Giannakakis A, Sandaltzopoulos R, Greshock J, Liang S, Huang J, Hasegawa K, Li C, O'Brien-Jenkins A, Katsaros D, Weber BL, et al: miR-210 links hypoxia with cell cycle regulation and is deleted in human epithelial ovarian cancer. Cancer Biol Ther 7: 255-264, 2008.

31. Bhattacharya R, Nicoloso M, Arvizo R, Wang E, Cortez A, Rossi S, Calin GA and Mukherjee P: miR-15a and miR-16 control Bmi-1 expression in ovarian cancer. Cancer Res 69: 9090-9095, 2009. 